\title{
A rational interaction: Lower bound on the number of I-PI graphs without tadpoles
}

\author{
G. A. T. F. DaCosta \\ Department of Mathernatics, University of Michigan, Ann Arbor, Michigan 48109
}

(Received 4 September 1990; accepted for publication 12 December 1990)

\begin{abstract}
A lower bound on the number of I-PI connected graphs without tadpoles that will be important in the proof of the divergence of the perturbation series of a model with rational interaction is obtained.
\end{abstract}

\section{INTRODUCTION}

In two previous papers we studied the perturbation series of a model with rational interaction of the form $\lambda^{2} \phi^{5}\left(1+\lambda \phi^{2}(x)\right)^{-1}$, in the context of quantum mechanics ${ }^{1}$ and quantum field theory in two dimensions. ${ }^{2}$ Here, we consider the same model as defined in Ref. 2 and this time we calculate a lower bound for the number of I-PI graphs that will be important in the proof of the divergence of the perturbation series of the model, to appear in a subsequent paper. There are two motivations for considering this interaction. First, models with interaction of the above type have aroused considerable interest due to their possible applications to laser theory (see the above references for more information on this). Second, they mimic some of the perturbation problems of renormalizable polynomial field theories like factorial growth of the number of diagrams, the presence of positive and negative signs mingled in the same order of perturbation, and divergence of the series which in most of the cases is very difficult to prove rigorously. ${ }^{3,4}$ The main difficulty resides in the fact that the subtractions of ultraviolet infinities are responsible for an oscillation of signs among the amplitudes belonging to the same order of perturbation that lead to cancellations. Prior to the calculation of a lower bound for the sum of these amplitudes therefore it is necessary to perform a careful analysis of which amplitude dominates which. The model we consider here, though, is supernormalizable. The only possible infinities are those associated to tadpole diagrams and they are eliminated from the perturbation series by Wick ordering the fields, after which we are left with positive and completely convergent amplitudes which are exponentially bounded. Although no renormalizations are necessary, there is an oscillation of signs inside the terms of the series but they are solely due to the rational form of the interaction. In our case this problem is a mild one since the way the signs appear can be complctely displayed from the outset. A harder problem concerns the counting of diagrams since we should count only those without tadpoles. In this paper we solve this counting problem.

\section{DIAGRAM COUNTING}

I-PI connected graphs are basically of two types: those whose vertices can be displayed forming a ring (we shall call them ring graphs) and therefore can be counted using Jaffe's method $^{3}$ and those whose vertices cannot. The latter are treelike graphs whose "lines" are ring graphs. A lower bound on their number can be achieved from (a bound on) the number $\gamma$ of ring graphs given by the following lemma.

Lemma 1: The number $\gamma(n)$ of connected I-PI ring graphs with $V=\Sigma V_{v}$ vertices contributing to order $n$ of the $E$-points Schwinger function of the two-dimensional model defined in Ref. 2 (as well as the symbols used here) is given by

$\gamma(n)=\prod_{v}\left(\begin{array}{l}v \\ 2\end{array}\right)^{V_{v}} \cdot 2^{V-1} \cdot(V-1) !\left(\begin{array}{c}2 n \\ E\end{array}\right) E !(2 n-E-1) ! !$

Proof: In a $v$-vertex (a vertex where $v$ fields meet) we can choose two fields in ( $\left.\stackrel{2}{2}_{2}^{\prime}\right)$ ways. If $V_{v}$ is the number of $\nu_{\text {- }}$ vertices, then $\mathrm{II}_{v}\left(\begin{array}{c}v^{v} \\ 2\end{array}\right)^{V_{v}}$ is the total number of ways of choosing two fields in each vertex. There are $2^{V-1} \cdot(V-1)$ ! ways to link the chosen fields in order to form a ring ( $V$ internal lines). Suppose all vertices are in a given order. Given one of them we can link one of the two fields chosen in two ways with the two fields of the next vertex in the order. The remaining field can be contracted in two ways with the third vertex in the order and so on. For a fixed order we have $2^{v-1}$ ways to form the circle. But we must consider all possible orders, i.e., $(V-1)$ !. There are $\left({ }^{\Sigma(v-2) V^{\prime}}\right)$ ways to choose $E$ fields among the $\Sigma(v-2) V_{v}=2 n \geqslant E$ in order to link them with $E$ external fields. $E$ ! contractions are possible. The other $I-V$ internal lines come from the

$$
\left(\sum(v-2) V_{v}-E-1\right) ! !=(2 n-E-1) ! !
$$

contractions of the rest of the fields.

Now, we classify the connected ring graphs into two types: those having tadpoles (lines with both ends hooked to the same vertex) and those not having them. The theorem below gives a unique expression that displays both types of graphs, but we will be able to decouple from it a much simpler formula that considers only graphs without tadpoles.

Remark: The making of the $I-V$ internal lines consists of forming

$$
\frac{1}{2} \sum(v-2) V_{v}-\frac{E}{2}=\#
$$

pairs of fields. By a formula given in the Appendix, they can be formed in 


$$
\begin{aligned}
& P\left(\sum_{v}(v-2) V_{v} \mid \#\right) \\
& =\left(\begin{array}{c}
\sum(v-2) V_{v} \\
E
\end{array}\right)(2 \#-1) ! ! \equiv P(\#)
\end{aligned}
$$

ways.

Theorem: Let $v$ be the number of fields hooked to a vertex and $P(\nu \mid p)$ the number of ways of forming $p$ tadpoles with them. Then $P(\#)$ is equal to

$$
\begin{aligned}
& \sum_{\Sigma(m,)_{1}=E} \prod_{t / 1}\left(\begin{array}{c}
\beta_{j}-2 \\
\left(m_{1}\right)_{\beta_{i}}
\end{array}\right) \\
& \times \prod_{\delta=1}^{\nu-2}\left\{\sum^{(a)} P\left(2 s_{\delta-1}+s_{2 \delta} \mid s_{2 \delta-1}\right) s_{2 \delta} !\right.
\end{aligned}
$$

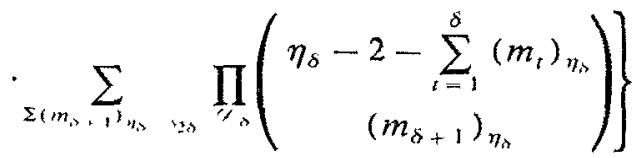

$$
\begin{aligned}
& \cdot \prod_{1,1} \sum_{2}^{(b)} P\left(\eta_{V-2}-2\right. \\
& \left.-\sum_{i=1}^{v-1}\left(m_{1}\right)_{\eta 1} \mid\left(s_{2 V-3}\right)_{\eta !}\right) s_{2 V-2} !
\end{aligned}
$$

In (3), the products II ", are over the elements $n_{s} \in \mathscr{Z}_{\delta}$ $: \mathscr{V}_{0}=\left\{\beta_{j}\right\}$ where $j=1,2, \ldots, V$ and $\mathscr{Q}_{\delta}=\left\{\beta_{\delta+1}, \ldots, \beta_{V}\right\}$. Sums $\Sigma^{(a)}$ and $\Sigma^{(b)}$ run over the possible values of $s_{2 \delta-1}, s_{2 \delta}$, and $\left(s_{2 r} \ldots{ }_{3}\right)_{\eta_{1}}, s_{2 v}, \sum_{2}$ satisfying the relations

$$
2 s_{2 \delta} \quad+s_{2 \delta}=\beta_{i}-2-\sum_{i=1}^{\delta}\left(m_{t}\right)_{\beta_{s}}
$$

and

$$
\left(s_{2 v-3}\right)_{\eta_{1}, 2}+s_{2 v-2}=\eta_{v-2}-\sum_{t=1}^{V_{-1}-1}\left(m_{t}\right)_{\eta_{1} \quad=},
$$

respectively. For $V=2$, consider (3) with $V=3$ but $\mathscr{Z}_{0}$ and $\mathscr{Z}$, having only two and one element, respectively.

Proof: An informal proof (for a formal one see the Appendix) is:

(a) Rewrite $\left({ }^{\Sigma\left(r_{2}-2\right) V_{1}}\right)$ so as to explicitly show all possible attachments of the external lines to the vertices. We have then

$$
\left(\begin{array}{c}
\Sigma(v-2) V_{v} \\
E
\end{array}\right)=\sum_{\Sigma_{j}\left(m_{1}\right\rangle_{k j,}=E} \prod_{v / 1}\left(\begin{array}{c}
\beta_{j-2} \\
\left(m_{1}\right)_{\beta_{1}}
\end{array}\right),
$$

where $0 \leqslant\left(m_{1}\right)_{\beta,} \leqslant \beta_{j}-2$ and

$$
\sum_{i}^{v}\left(\beta_{j}-2\right)=\sum(v-2) V_{v}
$$

obtained after application of the simplest case. The $\left(m_{1}\right)_{\beta_{2}}$ 's are nothing but the number of external lines hooked to the $\beta_{j}$ vertex.

(b) Given an attachment of the external lines pick one (any) of the vertices, e.g., the $\beta_{1}$ vertex where $\left(m_{1}\right)_{\beta_{1}}$ fields are already contracted with external ones and two fields link the vertex with other two. The remaining $\beta_{1}-2-\left(m_{1}\right)_{\beta_{1}}$ fields are to be contracted to form $s_{1}$ tadpoles and leave $s_{2}$ fields to be linked with those of other vertices. All the possibilities are comprised in the condition $2 s_{1}+s_{2}=\beta_{1}-2$ $-\left(m_{1}\right)_{\beta_{1}}$ (see Fig. 1). The sum $\Sigma^{(a)}$ in (3) runs over all the possible values $s_{1}$ and $s_{2}$ can assume. The second sum inside the brackets has the same meaning of that for the external lines. The $s_{2}$ lines coming from the $\beta_{1}$ vertex are seen as "external lines" by the other $V-1$ vertices and the sum is over all possible ways to hook these lines to those vertices. One should repeat this process until all vertices have been covered except two. The possible contractions among their fields are described by the sum $\Sigma^{(b)}$. Because the set $D_{r-2}$ has only two elements, we have a product of two $P$ 's that count for the tadpoles hung to each of the two vertices times $s_{2 v-2}$ ! possible contractions of the rest of the fields.

Now we take $s_{\sigma}=0$ for $\sigma$ odd to eliminate those graphs with tadpoles. Because $P(\cdot, 0) \equiv 1,(3)$ is reduced to the expression (7) below:

$$
\begin{aligned}
& \sum_{\Sigma\left(m_{0}\right)_{n_{1}}=E} \prod_{s_{0}}\left(\begin{array}{c}
\beta_{j}-2 \\
\left(m_{1}\right)_{\beta_{i}}
\end{array}\right)
\end{aligned}
$$

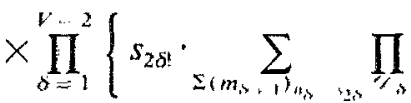

$$
\begin{aligned}
& \left.\times\left(\begin{array}{c}
\eta_{\delta}-2-\sum_{t=1}^{\delta}\left(m_{t}\right)_{\eta_{i}} \\
\left(m_{\delta+1}\right)_{\eta_{\phi}}
\end{array}\right)\right\} s_{2 V-2} !,
\end{aligned}
$$

where $s_{2 \diamond}$ is given by (8) with $s_{2 \sigma-1}=0$ and

$$
\begin{aligned}
s_{2 V-2} & =\beta_{V-1}-\sum_{t=1}^{r}\left(m_{t}\right)_{\beta_{i}}, \\
& =B_{V}-\sum_{i=1}^{r-1}\left(m_{t}\right)_{\beta_{i}} .
\end{aligned}
$$

When $V=2$ put $\delta=1$ and $s_{2 r_{-2}} !=1$.

Equation (7) can be bounded as follows. Using $\left(\begin{array}{l}c \\ b\end{array}\right) \geqslant 1$ we find that

$$
(7) \geqslant \prod_{\delta=1}^{V-1}\left(s_{2 \delta}\right) ! \geqslant 2^{-\Sigma \varepsilon_{2 \infty}}\left(\Sigma s_{2 \delta}\right) !
$$

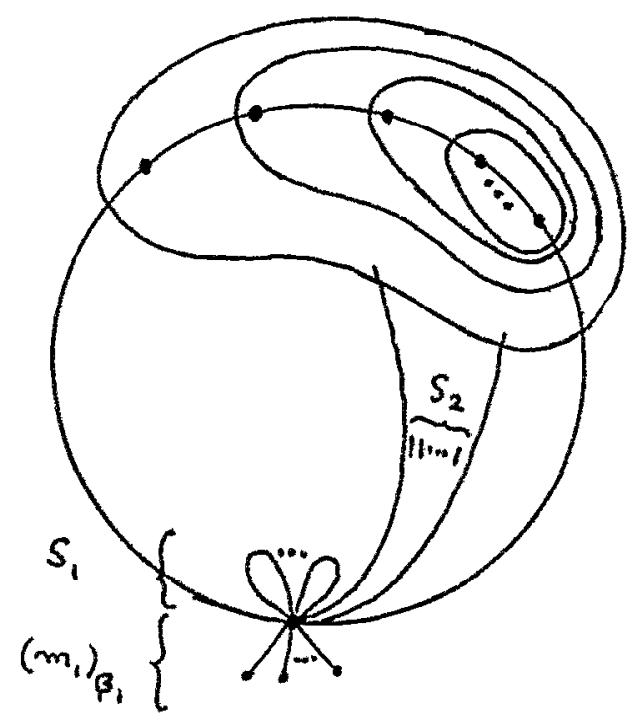

FIG. 1. Possibilities comprised in the condition $2 s_{1}+s_{2}=\beta_{1}$ $-2-\left(m_{1}\right)_{\beta_{1}}$. 
However one should not forget the conditions on the $m$ 's, namely,

$$
\begin{aligned}
& \sum_{j}\left(m_{1}\right)_{\beta_{1}}=E, \quad \beta_{j} \in \mathscr{D}_{0}, \\
& \sum\left(m_{\delta+1}\right)_{\eta_{n}}=\beta_{\delta}-2-\sum_{t-1}^{\delta}\left(m_{t}\right)_{\beta_{s}}, \\
& \eta_{\delta} \in \mathscr{D}_{\delta}, \quad \delta=1, \ldots, V-2, \\
& s_{2 v-2}=\beta_{v-1}-2-\sum_{t=1}^{V-1}\left(m_{t}\right)_{\beta_{v}}, \\
& =\beta_{V}-2-\sum_{t=1}^{V-1}\left(m_{t}\right)_{\beta_{i}} .
\end{aligned}
$$

When $\delta=V-2$ one can solve two of the above conditions for $\left(m_{1-1}\right)_{\beta_{1}}$ and $\left(m_{V}\right)_{\beta_{v}}$, to get

$$
\begin{aligned}
2 s_{2 V-2}= & \left(\beta_{V}-2\right)+\left(\beta_{V-1}-2\right)+\left(\beta_{V-2}-2\right) \\
& +\Sigma\left(m_{t}\right)_{\beta_{v} .}-\Sigma\left(m_{t}\right)_{\beta_{v},}-\Sigma\left(m_{t}\right)_{\beta_{V},},
\end{aligned}
$$

where $1 \leqslant t \leqslant V-2$. Adding up the $s_{2 \delta}$ 's, using (15) and the conditions $(10)$ one obtains that

$$
\sum_{\delta=1}^{V} s_{2 \delta}^{-1}=\frac{1}{2} \sum_{i}^{\nu}\left(\beta_{i}-2\right)-\frac{1}{2} \sum_{i}^{V}\left(m_{1}\right)_{\beta_{i}}=n-\frac{E}{2} .
$$

Therefore,

$$
\text { (7) }>22^{-n+E / 2} \cdot(n-E / 2) \text { ! . }
$$

We may conclude that the number of ring graphs without tadpoles, $\gamma_{w, t}$ is bounded from below by

$$
\gamma_{u, 1}>2^{V-1}(V-1) ! E ! 2^{-n+E / 2}(n-E / 2) ! .
$$

\section{ACKNOWLEDGMENTS}

I would like to thank Vincent Rivasseau for a critical reading of an earlier version of this paper. This work was supported by CNPq (Brazil).

\section{APPENDIX: A LEMMA AND A FORMAL PROOF OF THE THEOREM}

If $P(v \mid p)$ is the number of sets of $p$ pairs formed out of $v$ elements with no element in a pair appearing more than once within a set then

$$
P(v \mid p)=(2 p-1) ! !\left(\begin{array}{c}
v \\
v-2 p
\end{array}\right), \quad v \geqslant 2 p
$$

The number $P(v \mid p)$ satisfies the following relation

\section{Lemma:}

$$
\sum_{\substack{2 m_{1}+m_{1}=A \\ 2 m_{2}+m_{1}=B}} P\left(A \mid m_{1}\right) P\left(B \mid m_{2}\right) m_{3} !=(A+B-1) ! ! .
$$

Proof: One could apply Hermite polynomials to show (A.2). However, we rather prefer the following proof. Consider two vertices, each one with $A$ and $B$ fields hooked, respectively. The number of ways of linking these fields among themselves is $(A+B-1) ! !$. In general, this can be done by contracting $2 m_{1}$ fields of the $A$ vertex to form $m_{1}$ tadpoles. This can be accomplished in $P\left(A \mid m_{1}\right)$ manners leaving $m_{3}$ fields to be linked in $m_{3}$ ! ways with $m_{3}$ of the $B$ fields. Similarly, we can have $m_{2}$ tadpoles hanged in the $B$ vertex formed before the $m_{3}$ contractions, in $P\left(B \mid m_{2}\right)$ ways. If $2 m_{1}+m_{3}=A$ and $2 m_{2}+m_{3}=B$ then the sum in the lhs running over the possible values of $m_{1}, m_{2}$, and $m_{3}$ certainly covers all possible contractions and it equals the rhs.

Proceeding now to a proof of (3) we note that the set $\mathscr{D}_{v-2}$ has only two elements and $\eta_{v-2}$ can assume two values, say $\beta_{V-1}$ and $\beta_{V}$. Then by (A2) the last sum in (3) involving the two $P$ 's is equal to

$$
\begin{aligned}
\left(\sum_{V=2}\right. & {\left.\left[\eta_{V-2}-2-\sum_{t=1}^{v-1}\left(m_{t}\right)_{\eta_{V}}{ }_{2}\right]-1\right) ! ! } \\
= & \left(\sum_{v_{t=2}}\left[\eta_{V-2}-2-\sum_{t=1}^{V-2}\left(m_{t}\right)_{\eta_{t}} \quad=\right]\right. \\
& \left.-s_{2 V-4}-1\right) ! !
\end{aligned}
$$

We can then shift $(\mathrm{A} 3)$ to the left side of

$$
\begin{aligned}
& \left.\sum_{\Sigma\left(m_{V}\right.}\right)_{\eta_{V} \eta_{2}} \prod_{V=2}\left(\begin{array}{c}
\eta_{V-2}-2-\Sigma\left(m_{t}\right)_{\eta_{V}} \\
\left(m_{V-1}\right)_{\eta_{V}}
\end{array}\right) \\
& =s_{2} v+ \\
& =\left(\sum_{v=2} \begin{array}{c}
{\left[\eta_{V-2}-2-\Sigma\left(m_{t}\right)_{\eta_{V}}\right]} \\
s_{2 V-4}
\end{array}\right),
\end{aligned}
$$

where $1 \leqslant t \leqslant V-2$. By (A 1$)$,

$$
\begin{aligned}
(\mathrm{A} 2) \cdot(\mathrm{A} 3) & \\
= & P\left\{\sum_{V_{2}}\left(\eta_{V-2}-2-\sum_{t=1}^{v-2}\left(m_{t}\right)_{\eta_{t}}\right)\right. \\
& \left.\times \mid \frac{1}{2}\left[\sum_{i=2}\left(-\sum_{t=1}^{V-1} \cdot\right)\right]\right\} .
\end{aligned}
$$

By application of (A2), we can now obtain that

$$
\begin{gathered}
\sum P\left(\beta_{V-2}-2-\sum_{t=1}^{V-2}\left(m_{t}\right)_{\beta_{1},} \mid s_{2 V-5}\right) \cdot(\mathrm{A} 5) \cdot s_{2 V \cdot 4} ! \\
=\left(\sum_{V}\left[\eta_{V-3}-2-\sum_{i}^{V-2}\left(m_{t}\right)_{\eta_{V}, 3}\right]-1\right) ! !
\end{gathered}
$$

where the sum on the lhs of (A6) does satisfy the relations

$$
\begin{aligned}
& 2 s_{2 V-5}+s_{2 V-4}=\beta_{V-2}-2-\sum_{t=1}^{V-2}\left(m_{l}\right)_{\beta_{i}} \text {, , } \\
& 2\left[\frac{1}{2} \sum_{v=2}\left(\cdot-\sum_{i=1}^{v-1} \cdot\right)\right]+s_{2 v-4} \\
& =\sum_{v_{1}}\left(\eta_{V-2}-\sum_{1}^{V-2}\left(m_{t}\right)_{\eta_{v}}\right) \text {. }
\end{aligned}
$$

Proceeding as above one can exactly resum (3) to obtain

$$
\left(\sum_{j}(j-2) V_{j}-E-1\right) ! !
$$

'G. A. T. F. DaCosta and M. Gomes, "A non polynomial interaction: stability and Borel summability," Preprint.

'G. A. T. F. DaCosta and M. Gomes, J. Math. Phys. 30, 1007 (1989).

${ }^{3}$ A. Jaffe, Commun. Math. Phys. 1, 127 (1965).

${ }^{4}$ C. deCalan and V. Rivassesu, Commun. Math. Phys. 83, 77 (1982). 